Federal Reserve Bank of Minneapolis

Research Department

\title{
When should labor contracts be nominal?
}

\author{
Antoine Martin and Cyril Monnet* \\ Working Paper 603
}

July 2000

\begin{abstract}
This paper proposes a theory of when labor contract should be nominal or, instead, indexed. We find that, contracts should be indexed if prices are difficult to forecast and nominal otherwise. We use a principalagent model developed by Jovanovic and Ueda (1997), with moral hazard, renegotiation, and where a signal (the nominal value of the sales of the agent) is observed before renegotiation takes place. We show that their result, that the optimal contract is nominal when agents must choose pure strategies, is robust to the case where agents can choose mixed strategies in the sense that, for certain parameters, the optimal contract is still nominal. For other parameters, however, we show that the optimal contract is indexed. Our findings are consistent with two empirical regularities. First prices are more volatile with higher inflation and, second, countries with high inflation tend to have indexed contracts. Our theory suggests that it is because prices are difficult to forecast in high inflation countries that contracts are indexed.

*Martin, Federal Reserve Bank of Minneapolis and University of Minnesota; Monnet, Federal Reserve Bank of Minneapolis and University of Minnesota. We would like to thank Ed Green, Larry Jones and Narayana Kocherlakota for helpful comments. All remaining errors are ours. E-mail: amartin@econ.umn.edu, cyril@econ.umn.edu. The views expressed herein are those of the authors and not necessarily those of the Federal Reserve Bank of Minneapolis or the Federal Reserve System.
\end{abstract}




\section{Introduction}

In this paper, we build on a model proposed by Jovanovic and Ueda (1997) (JU) to provide an explanation for the fact that we observe both nominal and indexed labor contracts. We show that the theory is consistent with the empirical evidence that indexed contracts are more prevalent in high inflation countries than in low inflation ones.

JU propose an explanation for why we observe nominal contract. They use a principalagent model with moral hazard, renegotiation and a signal which is observed after the agent made his effort decision but before renegotiation takes place. The signal is the nominal value of the sales of the agent. They assume that the agent follows a pure strategy in choosing his effort level and show that although contract could depend on the real amount of sales of the agent (it could be indexed) it will, in fact, depend only on the signal (the contract is nominal). This is because indexed contracts are not renegotiationproof. The intuition for this goes as follows. Since the agent follows a pure strategy, the principal can infer, in equilibrium, the effort level that has been chosen. Assume that the principal offers an indexed contract. At the renegotiation stage, since the effort is sunk, the principal can offer a new contract that is independent of the real amount of sales. The agent, being risk-averse, will prefer such a contract, and so does the principal because it reduces her wage bill. Nominal contracts, on the other hand, are renegotiation-proof because they depend only on the signal which is observed before renegotiation takes place.

We modify JU's model and allow the agent to choose his effort level according to a mixed strategy. Under this hypothesis both nominal and indexed contracts can be renegotiation-proof because with mixed strategies the principal is not able to infer the agent's effort level anymore. We consider which type of contract is best for different parameter values and show that the contract will be indexed if the signal doesn't provide much information and will be nominal otherwise. The intuition for this result is that if the signal is sufficiently informative, it is not very expensive to provide incentives for the agent to choose the effort level preferred by the principal. The nominal value of sales is a good approximation to the real amount of sales which is an indication of the agents effort. If the signal is not good, the cost becomes very high because the signal doesn't reveal much about the agent's effort. Thus the principal prefers to offer an index 
contract that provides incentives at a lower cost.

Since the principal cares about the real amount of sales, the nominal value is most informative when prices are easy to forecast; so in this case contract should be nominal. On the other hand, when prices are difficult to forecast, which makes the signal not very informative, contracts should be indexed. This corresponds to our intuition and is consistent with two empirical regularities. First, there is an important literature (see Cukierman (1984) and the references therein) showing that higher inflation is accompanied by more volatile prices or, in other words, prices that are more difficult to predict. Second, countries with high inflation tend to have indexed contracts. Azariadis (1978) notes that: "Wage escalation is [...] widespread in countries which have recently experienced substantial inflationary episodes." ${ }^{2}$ For the postwar United-States, Holland (1995) has similar results. In particular, he finds that increases in inflation precede increases in wage indexation. In the light of these observations, our model suggests that the reason high inflation countries tend to have indexed contracts is because prices in these countries are difficult to forecast.

The literature related to our paper is fairly small. JU indicate that two reasons have been suggested for why contracts are nominal: "The price level cannot be observed in time (Lucas 1972) or is costly to incorporate into a contract (Dye 1981)." We are not aware of a paper that specifically considers the circumstances under which contracts should be nominal or indexed.

Azariadis-Cooper (1985a,b) and Cooper (1990) investigate economies for which there can be equilibria with nominal contracts as well as equilibria with indexed contracts. They incorporate ideas from the implicit contract literature (Azariadis (1975), Baily(1974)) into an overlapping generation model. Risk averse consumers whose consumption is risky when they are old can obtain insurance from risk neutral speculators who can sell them nominal claims. Azariadis-Cooper (1985a) show that the market for nominal claims will be open if consumers are sufficiently risk averse, and that the resulting equilibrium is constrained-optimal. Azariadis-Cooper (1985b) and Cooper (1990) introduce a government who prints money to finance its consumption and show that there are multiple equilibria. In particular, there is an equilibrium with nominal contracts if government

\footnotetext{
${ }^{2}$ See also the references in his paper.
} 
policy is not "too variable". Intuitively, this result is similar to ours. Freeman and Tabellini (1998) present a model in which nominal contracts can be optimal under certain assumptions. However they do not discuss when indexed or nominal contracts should be preferred. There is a literature concerned with the optimal degree of indexation of contracts (Gray (1976), Fischer(1977)) but in these models the form of the contract is given and is not derived from first principles.

The rest of the paper is built as follows. In section 2 we describe the model. Then, in section 3 , we present the problem of the principal. We find it convenient to consider three separate cases, the case when agents work hard with probability one, the case when they work hard with probability zero, and the case when they follow a mixed strategy. By comparing the utility of the principal in each case, we can determine the contract that will be chosen. In section 4 we present our main theorem and, in section 5, discuss when the principal wants to provide incentives for the agents to work hard with strictly positive probability. Section 6 concludes. 


\section{The model}

\subsection{Preferences}

The presentation of the model follows closely that of JU. We consider the case of an individual, the principal, who faces the problem of giving another individual, the agent, the incentive to make some work effort. The principal cannot observe the agent's effort but only a signal $s$ and then the output $y$. The level of effort influences the level of output through the probability of its occurence, $F_{e}(y) \equiv \operatorname{prob}($ output $\leq y \mid$ effort $=e$ ).

The agent has preferences over real income $w \in \mathbb{R}$ and effort $e \geq 0$ which can be represented by an additively separable utility function $U(w, e)=U(w)-\Psi(e)$. The function $U$ is defined on $(-\infty, \infty)$, is strictly increasing and strictly concave, and $\lim _{w \rightarrow-\infty} U(w)=-\infty, \lim _{w \rightarrow \infty} U(w)=\infty^{3}$. We impose no limits on the amount the principal can promise the agent. The function $\Psi: \mathbb{R}_{+} \rightarrow \mathbb{R}_{+}$denotes the cost of exerting a particular effort. Finally, we let $\underline{w}$ denote the reservation wage utility of the agent and define $u=U(\underline{w})$ the corresponding utility level.

The principal is risk neutral and she maximizes the difference between her expected revenue and her expected wage bill. We assume that the utility of the principal is zero if she chooses not to hire the agent.

\subsection{Signals}

The signal is the nominal value of the sales of the agent $s=P y$, where $P$ is the price level. The price level is eventually known by the principal before consumption takes place. This allows for the possibility of indexed contracts. Given a signal and an effort level, the price is distributed according to a non degenerate density function $g(P \mid s, e)$. Hence, upon observing a high signal, the principal does not know if the amount of sales was good and the price was low or if the amount of sales was bad and the price high.

\footnotetext{
${ }^{3}$ These assumptions are those made in Fudenberg and Tirole (1990)(FT) and allow a direct use of their theorems. As noted in their paper, most results hold for the case where the function $U$ is bounded below which allows limited liability for the agent.
} 


\subsection{The contract space}

Definition 2.1. An admissible compensation scheme is denoted by $c(e)$ and specifies the wage $w(P, s, e)$ as a function of the signal $s$ and price $P=s / y$, for each announced level of effort e.

Definition 2.2. An admissible contract, denoted by $c$, is a menu of admissible compensation scheme, one for each level of effort.

We let $\mathcal{C}$ denote the set of admissible contracts. The agent chooses an admissible compensation scheme from the contract offered by the principal. The compensation scheme specifies the income received by the agent for a given signal and a given state. Hence if the signal is $s$, the price is $P=s / y$ and the announced effort level is $e$, then the income received is $w(P, s, e)$. Because of strict concavity, each different level of income corresponds to a unique level of utility for the agent. Hence, we can think of a compensation scheme as specifying a level of utility, $U(w(P, s, e))$ for each signal and price observed and each announced level of effort. Abusing notation, we write the level of utility as $U(P, s, e)$.

The justification for our choice of $\mathcal{C}$ as the contract space are as in FT. On the one hand, letting the principal offer admissible compensation schemes that are conditional on the effort level announced by the agent allows her to screen the agent. On the other hand, we can appeal to the revelation principal to justify the use of a direct revelation mechanism where the agent report truthfully his effort level.

Definition 2.3. We call a contract nominal if $w(P, s, e)=w(s, e)$ for all $P$, and indexed otherwise.

A contract is nominal if the compensation schemes depend only on the signal and the announced level of effort. If $w(P, s, e) \neq w\left(P^{\prime}, s, e\right)$, for some $P$ and $P^{\prime}$, the compensation schemes depend also on the real outcome $y=s / P$. Given the signal, learning the level of prices is equivalent to learning the real outcome. Hence if $w(P, s, e) \neq w\left(P^{\prime}, s, e\right)$, for some $P$ and $P^{\prime}$, we can say that the compensation scheme depends both on the signal and on the price level, i.e. on the real output level $y$, and we call it an indexed contract. 


\subsection{Game and strategies}

The extensive form of the game is as follows. First the principal offers the agent a contract. If it is refused, the agent receives utility $u$ and the principal can not offer another contract. Next, if the contract has been accepted, the agent chooses an effort strategy and determines the effort level. After the effort has been made, the signal is observed. Then, at the renegotiation stage the principal can choose to offer the agent a new contract. If it is not accepted the original contract remains valid and the principal can not offer another one. Next, the agent chooses a compensation scheme from the feasible contract. Finally the outcome of the project is observed and payments are made. We include the time line in the Appendix.

Definition 2.4. A contract is renegotiation-proof if, given the realized signal, there is no other contract that Pareto dominates it at the renegotiation stage.

A strategy for the agent is a function with domain $\mathbb{R}_{+} \times \mathcal{C}$ and range $[0,1]$. Given an admissible contract, the agent chooses a probability function $\mu: \mathbb{R}_{+} \rightarrow[0,1]$ which assigns probability $\mu(e)$ of exerting effort level $e$ and $\int_{0}^{\infty} \mu(e) d e=1$.

A strategy for the principal is a choice of a renegotiation-proof contract, given the strategy of the agent. We study the problem of the principal in the next section.

\section{The principal's problem}

In the remainder of the paper we consider the case where there are two possible signals, $H$ and $L$ as well as two levels of real outcomes $y_{H}$ and $y_{L}$ and two levels of effort $\bar{e}$ and $\underline{e}$. We assume $H>L, y_{H}>y_{L}$ and $\bar{e}>\underline{e}$. The distribution functions become discrete probability measures, but we keep the same notation. Therefore, $F_{e}(\sigma) \equiv \operatorname{prob}(s=\sigma \mid e)$ for $\sigma \in\{H, L\}$. For simplicity, we abuse notation and write $g\left(y_{H} \mid s, e\right)=g\left(s / y_{H} \mid s, e\right) \equiv \operatorname{prob}\left(P=s / y_{H} \mid s, e\right)$. Therefore we use $g$ interchangeably to denote the probability measure for the real output and for the price level ${ }^{4}$. Moreover we use $g(y \mid e)=\sum_{s} g(y \mid s, e) F_{e}(s)$ as the probability of being in state $y$ if the agent chose a

\footnotetext{
${ }^{4}$ We can do this because given $P$ and $s$ we know the real output level $y=s / P$ and given $y$ and $s$ we also know the price level $P=s / y$.
} 
level of effort $e$. The cost of effort function $\Psi$ is such that $\Psi(\underline{e})=0$ and $\Psi(\bar{e})=\Psi>0$.

To fix ideas, consider the following example. Assume the price level $P$ can take three values, $P_{1}<P_{2}<P_{3}$ and the real amount $y$ of sales is either $y_{H}$ or $y_{L}$. Let $P_{1} y_{H}=P_{2} y_{L}=L, P_{2} y_{H}=P_{3} y_{L}=H$ and assume that $\operatorname{prob}\left(P=P_{3}\right.$ and $\left.y=y_{H}\right)=$ $\operatorname{prob}\left(P=P_{1}\right.$ and $\left.y=y_{L}\right)=0$. Hence, upon observing a high signal, the principal does not know if the amount of sales was good and the price was low or if the amount of sales was bad and the price medium.

Assumption 3.1. $F_{\bar{e}}(H)>F_{\underline{e}}(H)$ and $g\left(y_{H} \mid H, \bar{e}\right)>g\left(y_{H} \mid H, \underline{e}\right)$.

The first inequality says that the high signal is more likely to occur if the high effort has been chosen, while the second says that conditional on the high signal, the good state is more likely when the high effort has been chosen. Notice that, from probability theory, $g\left(y_{H} \mid e\right)=F_{e}(H) g\left(y_{H} \mid H, e\right)+F_{e}(L) g\left(y_{H} \mid H, e\right), e \in\{\bar{e}, \underline{e}\}$, so our assumptions imply $g\left(y_{H} \mid \bar{e}\right)>g\left(y_{L} \mid \underline{e}\right)$.

We now describe the problem of the principal when she wants to induce effort with a strictly positive probability. Let $\mu$ denote the probability with which the agent chooses the high effort. We let $\mathcal{R} \mathcal{P}$ be the set of renegotiation-proof contracts. These are the contract that can not be Pareto-improved upon at the renegotiation stage. A point in $\mathcal{R P}$ is formed of a probability $\mu \in[0,1]$ and of eight promised utility levels $\{U(y, s, e)\}$ for each $s \in\{H, L\}, y \in\left\{y_{H}, y_{L}\right\}$ and $e \in\{\bar{e}, \underline{e}\}$. Hence, we can write $\mathcal{R} \mathcal{P}$ as:

$$
\mathcal{R P}=\left\{\begin{array}{c}
\mu,\{U(y, s, e)\} \text { for all } s, y, e \text { such that: } \\
\{U(y, s, e)\} \text { for all } s, y, e \text {, is renegociation-proof }
\end{array}\right\} .
$$

We will characterize such contracts below.

The principal solves the following maximization problem $(M P)$ :

$$
\underset{\mu,\{w(y, s, e)\}}{\operatorname{Max}} \sum_{e=\bar{e}, \underline{e}} \mu(e)\left\{\sum_{s=H, L} F_{e}(s)\left[\sum_{y=y_{H}, y_{L}} g(y \mid s, e)(y-w(y, s, e))\right]\right\}
$$

subject to the following constraints ${ }^{5}$

(a) the ex-ante incentive compatibility constraint (IC),

\footnotetext{
${ }^{5}$ The constraints are written explicitly in the appendix.
} 
(b) the individual rationality constraint (IR),

(c) the fact that $\mu$ must be a best response for the agent and

(d) the contract is renegotiation-proof.

As stated previously, the objective function of the principal is the expected revenue minus the expected wage bill. The ex-ante incentive compatibility constraint says that the agent must get at least as much utility from choosing the high effort than from choosing the low effort and exactly the same utility if he randomizes. The other constraints are standard.

Instead of trying to solve this problem directly, it will prove useful to consider two different cases, $\mu=1$ and $\mu \in(0,1)$. It is also useful to find the utility of the principal when she doesn't provide any incentives for the agent to work hard, so we will also look at the case where $\mu=0$. As we show below, the contract takes, in each of these cases, a simple form. Then, for a given set of parameters, we can compare the value the principal gets from the contract in each case and determine which one is best for her.

\subsection{Case I: $\mu=0$}

Here, the principal does not provide the agent with any incentive to exert effort, thus the wage offered is the reservation wage $\underline{w}$ and the utility of the principal, denoted $V_{0}$, is given by

$$
V_{0}=y_{L}+g\left(y_{L} \mid \underline{e}\right) \Delta-\underline{w},
$$

where $\Delta \equiv y_{H}-y_{L}$.

This contract is renegotiation-proof.

\subsection{Case II: $\mu=1$}

Now the principal wants the agent to always exert high effort. We know from the renegotiation-proofness condition that $U(y, H, \bar{e})=U(H)$ and $U(y, L, \bar{e})=U(L)$ for all $y$, and $U(s, y, \underline{e})=u$ for all $s$ and $y$. The intuition is that when $\mu=1$, any contract that has $U\left(y_{H}, s, e\right) \neq U\left(y_{L}, s, e\right)$, for some $s$ and $e$, is not renegotiation-proof. Since the 
utility levels offered in this contract are independent of the real amount of sales of the agent $y$, we call it a nominal contract. Notice that, since the signal is observed before renegotiation takes place, nominal contract are renegotiation-proof.

Combining the (IC) and (IR) constraints yields a unique solution to the principal problem,

$$
\begin{aligned}
U(H) & =u+\Psi\left[\frac{1-F_{\underline{e}}(H)}{F_{\bar{e}}(H)-F_{\underline{e}}(H)}\right], \\
U(L) & =u-\Psi\left[\frac{F_{\underline{e}}(H)}{F_{\bar{e}}(H)-F_{\underline{e}}(H)}\right] .
\end{aligned}
$$

Under this contract, the agent receives an amount that depends on the signal, the nominal value of his sales, but not on the real amount of those sales, and his expected utility is $u+\Psi$. Note that $U(H)-U(L)$ increases with $\Psi$ and decreases with $F_{\bar{e}}(H)-$ $F_{\underline{e}}(H)$. The payoff of the agent from offering this contract will be denoted by $V_{1}$ and is given by

$$
V_{1}=y_{L}+g\left(y_{H} \mid \bar{e}\right) \Delta-W_{1},
$$

where $W_{1}=F_{\bar{e}}(H) w(H)+\left(1-F_{\bar{e}}(H)\right) w(L)$ is the expected wage bill of the principal under this contract. $w(s)$ is such that $U(w(s))=U(s)$ for $s \in\{H, L\}$.

The following technical lemma will be used in the proof of our main theorem. All proofs are in the appendix.

Lemma 3.2. Given $F_{\underline{e}}(H), W_{1} \longrightarrow \infty$ as $F_{\bar{e}}(H) \longrightarrow F_{\underline{e}}(H)$.

\subsection{Case III: $\mu \in(0,1)$}

In this case, the contract offered by the principal looks a lot like the one derived by FT. It is a little more complicated because we have a signal. However, it is easy to prove that if the signal is uncorrelated with the real outcome, the contracts in our model and in FT are exactly the same. For a given signal, and a given $\mu$, the form of the renegotiation-proof contract is given by lemma 2.1 (p. 1284) in FT. We restate it here, using our notation. 
Lemma 3.3. (FT, Lemma 2.1) Given a signal s, if $c=\left\{U\left(y_{H}, s, e\right), U\left(y_{L}, s, e\right)\right\}_{e \in\{\bar{e}, \underline{e}\}}$ is a renegotiation-proof contract for distribution $\mu$, then

either

$$
g\left(y_{H} \mid \underline{e}\right) U\left(y_{H}, s, \underline{e}\right)+g\left(y_{L} \mid \underline{e}\right) U\left(y_{L}, s, \underline{e}\right)>g\left(y_{H} \mid \bar{e}\right) U\left(y_{H}, s, \bar{e}\right)+g\left(y_{L} \mid \bar{e}\right) U\left(y_{L}, s, \bar{e}\right)
$$

so that, at the renegotiation stage, the expected utility of an agent who chose low effort is strictly higher than the expected utility of an agent who chose high effort, or the following three conditions hold:

1. $U\left(y_{H}, s, \underline{e}\right)=U\left(y_{L}, s, \underline{e}\right)=u$,

2. $U\left(y_{H}, s, \bar{e}\right) \geq U\left(y_{L}, s, \bar{e}\right)$,

3. $g\left(y_{H} \mid s, \underline{e}\right) U\left(y_{H}, s, \underline{e}\right)+g\left(y_{L} \mid s, \underline{e}\right) U\left(y_{L}, s, \underline{e}\right)=g\left(y_{H} \mid s, \underline{e}\right) U\left(y_{H}, s, \bar{e}\right)+g\left(y_{L} \mid s, \underline{e}\right) U\left(y_{L}, s, \bar{e}\right)$.

so that, at the renegotiation stage, the incentive compatibility constraint is binding for agents who chose low effort.

Under the first type of contracts, an agent will never choose to work hard, so we consider only the second type of contracts. Since the conditions (1) to (3) must hold for any signal $s$, we have in particular that $U(y, H, \underline{e})=U(y, L, \underline{e})=u$, for $s \in\{H, L\}$. To be consistent with FT, we call condition (3) the interim incentive compatibility constraints. Notice that the last constraints do not include the cost of exerting high effort since it is sunk at the renegotiation stage.

When there is no signal, as in FT, the constraint set yields a singleton, but it is not true here. In this case, we know that four constraint must hold with equality, the interim incentive compatibility constraint, for each signal, the ex-ante incentive compatibility constraint and the individual rationality constraint. Using lemma 3.3 , we can see that the last two constraints are equivalent and we are left with:

$$
\begin{aligned}
u= & g\left(y_{H} \mid H, \underline{e}\right) U\left(y_{H}, H, \bar{e}\right)+g\left(y_{L} \mid H, \underline{e}\right) U\left(y_{L}, H, \bar{e}\right) \\
u= & g\left(y_{H} \mid L, \underline{e}\right) U\left(y_{H}, L, \bar{e}\right)+g\left(y_{L} \mid H, \underline{e}\right) U\left(y_{L}, L, \bar{e}\right) \\
\Psi+u= & F_{\bar{e}}(H)\left[g\left(y_{H} \mid H, \bar{e}\right) U\left(y_{H}, H, \bar{e}\right)+g\left(y_{L} \mid H, \bar{e}\right) U\left(y_{L}, H, \bar{e}\right)\right]+ \\
& F_{\bar{e}}(L)\left[g\left(y_{H} \mid L, \bar{e}\right) U\left(y_{L}, L, \bar{e}\right)+g\left(y_{L} \mid L, \bar{e}\right) U\left(y_{L}, L, \bar{e}\right)\right] .
\end{aligned}
$$


These three equations in four unknowns give us a family of solution that we can parameterize by one of the unknowns. We let $Z=U\left(y_{H}, L, \bar{e}\right)$ and then express all the other utility levels in terms of $Z$. We get

$$
\begin{aligned}
U\left(y_{L}, L, \bar{e}\right) & =\frac{u}{g\left(y_{L} \mid L, \underline{e}\right)}-\frac{g\left(y_{L} \mid L, \underline{e}\right)}{g\left(y_{L} \mid L, \underline{e}\right)} Z, \\
U\left(y_{H}, H, \bar{e}\right) & =\frac{1-g\left(y_{H} \mid H, \underline{e}\right)}{g\left(y_{H} \mid H, \bar{e}\right)-g\left(y_{H} \mid H, \underline{e}\right)} \chi(Z), \\
U\left(y_{L}, H, \bar{e}\right) & =\frac{u}{g\left(y_{L} \mid H, \underline{e}\right)}-\frac{g\left(y_{H} \mid H, \underline{e}\right)}{g\left(y_{H} \mid H, \bar{e}\right)-g\left(y_{H} \mid H, \underline{e}\right)} \chi(Z) .
\end{aligned}
$$

The expression for $\chi(Z)$ is given in the appendix, where we also show how to obtain $\mu$ as a function of $Z$. The utility levels offered in this contract depend both on the signal and the real amount of sale. Given the nominal value of the sales, knowing the real amount is equivalent to knowing the price level. Thus we can think of this contract as depending on the signal and the level of prices. This is the reason why we call it an indexed contract.

The problem of the principal then reduces to choosing the value of $Z$, denoted $Z^{*}$ that maximizes her profit ${ }^{6}$. We let $V_{\mu}\left(Z^{*}\right)$ denote the maximum value of the objective function and we have

$$
\begin{aligned}
V_{\mu}\left(Z^{*}\right)= & y_{L}+\Delta\left[g\left(y_{H} \mid \underline{e}\right)+\mu\left(Z^{*}\right)\left(g\left(y_{H} \mid \bar{e}\right)-g\left(y_{H} \mid \underline{e}\right)\right)\right] \\
& -\mu\left(Z^{*}\right) W_{\mu}\left(Z^{*}\right)-\left(1-\mu\left(Z^{*}\right)\right) \Phi(u),
\end{aligned}
$$

where, $W_{\mu}$ is the expected wage bill of the principal if the agent chooses the high effort. It is given by

$$
\begin{aligned}
W_{\mu}= & F_{\bar{e}}(H)\left[g\left(y_{H} \mid H, \bar{e}\right) w\left(y_{H}, H, \bar{e}\right)+g\left(y_{L} \mid H, \bar{e}\right) w\left(y_{L}, H, \bar{e}\right)\right] \\
& +F_{\bar{e}}(L)\left[g\left(y_{H} \mid L, \bar{e}\right) w\left(y_{H}, L, \bar{e}\right)+g\left(y_{H} \mid L, \bar{e}\right) w\left(y_{H}, L, \bar{e}\right)\right]
\end{aligned}
$$

Where $w(y, s, e)$ is such that $U(w(y, s, e))=U(y, s, e)$. Notice that the value of $y_{L}$ has no effect on the type of contract the principal chooses to offer. Thus we can assume that $y_{L}$ is high enough so that the principal always prefers to offer the agent a contract.

We will need two other lemma for the proof of our main theorem.

\footnotetext{
${ }^{6}$ This $Z$ exists as the principal maximizes a continuous objective function over a compact set.
} 
Lemma 3.4. For any $\varepsilon>0$, there exists $\mu>0$ small enough such that $V_{\mu}-V_{0}<\varepsilon$.

This lemma states that as $\mu$ tends to zero, the value to the principal of offering an indexed contract tends to something equal to $V_{0}$ or lower.

Lemma 3.5. There exists $\bar{\Delta}>0$ big enough such that $V_{1}>V_{0}$ for all $\Delta \geq \bar{\Delta}$.

$\Delta$ is the gross surplus from getting the high output. Therefore, for some high enough surplus, the principal always prefers to have the agent working hard.

In this section, we have derived the value to the principal of offering a contract for three cases, 1) $\mu=0,2) \mu=1$, and 3) $\mu \in(0,1)$. For a given set of parameters, we can determine the case in which the principal gets the highest utility and thus find what type of contract she will offer.

\section{Indexed vs. nominal contracts}

In this section, we show our main result: the principal will choose indexed contracts whenever the signal does not give much information on the effort level chosen by the agent and she will choose nominal contracts otherwise.

Let $\mathcal{P}$ denote a choice of parameters for our model, $\mathcal{P}=\left\{\underline{w}, \Psi, y_{H}, y_{L}\right\}$. Let $V_{1}^{\sigma}(\mathcal{P})$ denote the value to the principal of a contract if $\mu=1, \mathcal{P}$ is the choice of parameters, and $F_{\bar{e}}(H)-F_{\underline{e}}(H)=\sigma$. Note that, everything else being equal, $V_{1}^{\sigma}(\mathcal{P})<V_{1}^{\sigma^{\prime}}(\mathcal{P})$ if $\sigma^{\prime}>\sigma$.

\section{Theorem 4.1.}

(1) Given $\mathcal{P}$ and any $\delta>0$. Assume that $F_{\bar{e}}(H), g(y \mid s, \bar{e})$, all $y, s, g\left(y_{L} \mid s, \underline{e}\right)$, all $s$, as well as the difference $g\left(y_{H} \mid H, \bar{e}\right)-g\left(y_{H} \mid H, \underline{e}\right)$ belong to $[\delta, 1]$. Then there is $\epsilon(\mathcal{P})>0$ such that:

If $F_{\bar{e}}(H)-F_{\underline{e}}(H)<\epsilon(\mathcal{P})$ then $V_{\mu}>V_{1}$.

(2) Let $\delta>0$. Assume that $F_{\bar{e}}(H)-F_{\underline{e}}(H)$ belongs to $[\delta, 1]$ and $V_{1}^{\delta}\left(\mathcal{P}^{\prime}\right)>V_{0}$. Then there is $\epsilon^{\prime}\left(\mathcal{P}^{\prime}\right)>0$ such that:

If $g\left(y_{H} \mid H, \bar{e}\right)-g\left(y_{H} \mid H, \underline{e}\right)<\epsilon^{\prime}\left(\mathcal{P}^{\prime}\right)$ then $V_{1}>V_{\mu}$. 
Notice that, under (1), the probabilities $F_{\bar{e}}(H)$ and $g(y \mid s, \bar{e})$, all $y, s, g\left(y_{L} \mid s, \underline{e}\right)$, all $s$, and the difference $g\left(y_{H} \mid H, \bar{e}\right)-g\left(y_{H} \mid H, \underline{e}\right)$ are not fixed. As we change $F_{\bar{e}}(H)-F_{\underline{e}}(H)$ they are allowed to change as long as they remain in the interval $[\delta, 1]$. A similar remark hold for (2).

This theorem simply says that as $F_{\bar{e}}(H) \longrightarrow F_{\underline{e}}(H)$, the cost for the principal of offering a nominal contract goes to infinity. The restrictions we impose on the other probability in our theorem assure that the cost of offering an indexed contract is bounded. This is why, as $F_{\bar{e}}(H)-F_{\underline{e}}(H)$ gets smaller, the principal will eventually prefer to choose an indexed contract. When the effort level has little effect on the realization of the signal and the agent wage is tied to the signal only, the agent doesn't have much incentive to work hard since the increase in his expected income (and thus expected utility) is small. In order to increase the incentives, the principal must increase the promised utility level for a good signal. This gets more expensive as the effect of effort on the signal gets smaller. The logic for the other case is very similar. As $g\left(y_{H} \mid H, \bar{e}\right) \longrightarrow g\left(y_{H} \mid H, \underline{e}\right)$, the cost for the principal of offering an indexed contract goes to infinity, and thus the principal chooses $\mu$ smaller and smaller and eventually, the principal prefers to choose $V_{1}$ rather than $V_{\mu}$.

We want to show that this theorem supports the intuition that indexed contracts are used when the signal does not provides much information and non-indexed contracts are used otherwise. To do that, we use Bayes rule and write

$$
F \bar{e}(H)-F_{\underline{e}}(H)=\frac{F(H)}{\mu(1-\mu)}[g(\bar{e} \mid H)-\mu] .
$$

where $F(H)=\mu F_{\bar{e}}(H)+(1-\mu) F_{\underline{e}}(H)$. By definition, $g(\bar{e} \mid H)-\mu$ is the marginal information provided by the high signal. Hence, if this information is small enough, $F_{\bar{e}}(H)-F_{\underline{e}}(H)$ will be small and, by theorem 4.1, indexed contracts are preferred.

Similarly, using Bayes rule we have

$$
g\left(y_{H} \mid H, \bar{e}\right)-g\left(y_{H} \mid H, \underline{e}\right)=\frac{g(\underline{e}, H) F(H)}{F_{\bar{e}}(H)(F(H)-g(\underline{e}, H))}\left[g\left(\bar{e} \mid y_{H}, H\right)-g(\bar{e} \mid H)\right] .
$$

By definition, $g\left(\bar{e} \mid y_{H}, H\right)-g(\bar{e} \mid H)$ is the marginal information provided by the realization of the good real sales, given that the high signal was observed. If this information is very 
small, the principal doesn't expect to learn much by observing the real state and prefers to propose a nominal contract.

We expect a nominal contract to be most informative in the case where prices are easy to forecast. Indeed, if prices can be forecasted perfectly, the nominal signal reveals all the relevant information. At the other extreme, the signal might be pure noise and reveal absolutely no information. We thus expect to see nominal contracts when prices are easy to forecast and indexed contracts when they are not. As mentioned earlier, the conclusions of our model are remarkably consistent with two empirical regularities. First it is recognized that higher inflation makes prices more difficult to forecast and, second, countries experiencing high inflation tend to have more indexed contracts. What our model suggests is that indexed contracts are more prevalent in high inflation countries because prices are hard to predict.

It is also interesting to note that our model suggests a way through which inflation can have real effects. Indeed, an increase in inflation may lead to a switch from nominal contracts to indexed contracts. This in turn has an impact on the effort exerted by the agent. In our model, the expected output is lower under indexed contracts because the agent chooses the high effort with a smaller probability than under nominal contracts. This however might not be true in a more general model. Consider, for example, a model with three levels of effort, high, medium and low. Suppose the level of effort chosen with probability 1 under nominal contract is the medium level. Under an indexed contract, the agent might randomize between the three level of efforts and consequently expected output could be higher than under the nominal contract if high effort is chosen with high probability.

\section{When Should the Agent Work Hard?}

In this section, we determine loose parameters regions where the principal wants the agent to exert the high level of effort with strictly positive probability. That is we characterize some situations where either $V_{0}>\max \left\{V_{1}, V_{\mu}\right\}$ or where $\min \left\{V_{1}, V_{\mu}\right\}>V_{0}$. The parameters we are mostly interested in are the net gain from being in the good rather than the bad state, $\Delta$ and the cost of exerting the high effort suffered by the agent $\Psi$. 
Note here that once we know that $V_{0}>\max \left\{V_{1}, V_{\mu}\right\}$ or that $\min \left\{V_{1}, V_{\mu}\right\}>V_{0}$, theorem 1 applies so that we can find regions where $V_{1}>V_{\mu}$ or where $V_{1}<V_{\mu}$.

It is easy to see (the proofs are provided in the Appendix) that if $\Delta$ is very small, the principal will not be willing to give any incentive for the agent to work hard. Furthermore, if the cost $\Psi$ of hard work is very high, then once again the principal will not give any incentive for hard work. Intuitively, if the cost of hard work is too high, it will be too expensive for the principal to elicit effort and she will choose not to do it. To summarize, we have $\min \left\{V_{1}, V_{\mu}\right\}>V_{0}$ for $\Delta$ high enough or for $\Psi$ small enough. $V_{0}>V_{1}$ and, for any $\varepsilon>0, V_{\mu}-V_{0}<\varepsilon$ otherwise.

\section{Conclusion}

In this paper we proposed an explanation of why we observe both indexed and nominal contract. We modified a principal-agent model proposed by Jovanovic-Ueda (1997), with moral hazard, renegotiation and where a signal (the nominal value of the sales of the agent) is observed before renegotiation takes place. Our model relaxes an assumption made by JU that agents choose pure strategies. We are able to show that their explanation for the occurrence of nominal contract is robust to this change since, for some parameters, the optimal contract is nominal in our model. We also show that, for other parameters, the optimal contract is indexed.

In order to keep the analysis tractable, we have chosen to consider a very simple model where the agent can choose a high or a low level of effort, the signal can only take two values, as can the real amount of sales of the agent. However, we do not believe that our results would be substantially altered by considering a more general setup.

We proved that, according to this theory, contracts should be indexed when the signal is not very informative and nominal otherwise. Since the signal, in this model, is the nominal value of the sale of the agent, it will not be very informative when the prices are difficult to forecast. Our result is consistent with empirical regularities, first higher inflation makes prices more difficult to forecast and, second, countries having high inflation tend to have indexed contracts. The theory thus suggests that because the signal will be less informative in countries with high inflation, they will choose to have 
indexed contracts. Similarly, in countries where inflation is low, the signal will carry enough information and the contracts will be nominal. 


\section{Appendix}

In this Appendix, for convenience, we let $\Phi(U)$ be the inverse function corresponding to $U$, such that $\Phi(U(w))=w$, all $w \in \mathbb{R}$.

\subsection{Constraints for the problem of the principal}

$$
\begin{aligned}
(I C) & F_{\bar{e}}(H)\left[g\left(y_{H} \mid H, \bar{e}\right) U\left(y_{H}, H, \bar{e}\right)+g\left(y_{L} \mid H, \bar{e}\right) U\left(y_{L}, H, \bar{e}\right)\right] \\
& +F_{\bar{e}}(L)\left[g\left(y_{H} \mid L, \bar{e}\right) U\left(y_{H}, L, \bar{e}\right)+g\left(y_{L} \mid L, \bar{e}\right) U\left(y_{L}, L, \bar{e}\right)\right]-\Psi(\bar{e}) \\
& \geq F_{\underline{e}}(H)\left[g\left(y_{H} \mid H, \underline{e}\right) U\left(y_{H}, H, \underline{e}\right)+g\left(y_{L} \mid H, \underline{e}\right) U\left(y_{L}, H, \underline{e}\right)\right] \\
& +F_{\underline{e}}(L)\left[g\left(y_{H} \mid L, \underline{e}\right) U\left(y_{H}, L, \underline{e}\right)+g\left(y_{L} \mid L, \underline{e}\right) U\left(y_{L}, L, \underline{e}\right)\right]
\end{aligned}
$$

$(I R)$

$$
\begin{aligned}
& F_{\bar{e}}(H)\left[g\left(y_{H} \mid H, \bar{e}\right) U\left(y_{H}, H, \bar{e}\right)+g\left(y_{L} \mid H, \bar{e}\right) U\left(y_{L}, H, \bar{e}\right)\right] \\
& +F_{\bar{e}}(L)\left[g\left(y_{H} \mid L, \bar{e}\right) U\left(y_{H}, L, \bar{e}\right)+g\left(y_{L} \mid L, \bar{e}\right) U\left(y_{L}, L, \bar{e}\right)\right]-\Psi(\bar{e}) \geq u
\end{aligned}
$$

$(B R) \quad \mu(\bar{e}) \in \operatorname{argmax}$

$$
\begin{aligned}
& \mu\left\{F_{\bar{e}}(H)\left[g\left(y_{H} \mid H, \bar{e}\right) U\left(y_{H}, H, \bar{e}\right)+g\left(y_{L} \mid H, \bar{e}\right) U\left(y_{L}, H, \bar{e}\right)\right]\right. \\
&\left.+F_{\bar{e}}(L)\left[g\left(y_{H} \mid L, \bar{e}\right) U\left(y_{H}, L, \bar{e}\right)+g\left(y_{L} \mid L, \bar{e}\right) U\left(y_{L}, L, \bar{e}\right)\right]-\Psi(\bar{e})\right\} \\
&+(1-\mu)\left\{F_{\underline{e}}(H)\left[g\left(y_{H} \mid H, \underline{e}\right) U\left(y_{H}, H, \underline{e}\right)+g\left(y_{L} \mid H, \underline{e}\right) U\left(y_{L}, H, \underline{e}\right)\right]\right. \\
&\left.\left.+F_{\underline{e}}(L)\left[g\left(y_{H} \mid L, \underline{e}\right) U\left(y_{H}, L, \underline{e}\right)+g\left(y_{L} \mid L, \underline{e}\right) U\left(y_{L}, L, \underline{e}\right)\right]\right\}\right\} \\
&(R P) \quad\left(\mu,\left\{U\left(y_{H}, s, e\right), U\left(y_{L}, s, e\right)\right\}_{s \in\{H, L\}}\right) \in \mathcal{R} \mathcal{P}
\end{aligned}
$$

\subsection{Parametrization of the indexing contract}

1. $U\left(y_{H}, L, \bar{e}\right)=Z$

2. $U\left(y_{L}, L, \bar{e}\right)=\frac{u}{g\left(y_{L} \mid L, \underline{e}\right)}-\frac{g\left(y_{H} \mid L, \underline{e}\right)}{g\left(y_{L} \mid L, \underline{e}\right)} Z$

3. $U\left(y_{H}, H, \bar{e}\right)=\frac{1-g\left(y_{H} \mid H, \underline{e}\right)}{g\left(y_{H} \mid H, \bar{e}\right)-g\left(y_{H} \mid H, \underline{e}\right)} \chi(Z)$

4. $U\left(y_{L}, H, \bar{e}\right)=\frac{u}{g\left(y_{L} \mid H, \underline{e}\right)}-\frac{g\left(y_{H} \mid H, \underline{e}\right)}{g\left(y_{H} \mid H, \bar{e}\right)-g\left(y_{H} \mid H, \underline{e}\right)} \chi(Z)$ 
5. $\mu=\min \left\{\mu_{H}, \mu_{L}\right\}$

6. $\mu(s)=\frac{\Phi^{\prime}(u) \frac{g\left(y_{H} \mid s, \bar{e}\right)-g\left(y_{H} \mid s, \underline{e}\right)}{g\left(y_{H} \mid s, \bar{e}\right) g\left(y_{L} \mid s, \bar{e}\right)}}{\Phi^{\prime}(U(s, g, \bar{e})(Z))-\Phi^{\prime}(U(s, b, \bar{e})(Z))+\Phi^{\prime}(u) \frac{g\left(y_{H} \mid s, \bar{e}\right)-g\left(y_{H} \mid s, \underline{e}\right)}{g\left(y_{H} \mid s, \bar{e}\right) g\left(y_{L} \mid s, \bar{e}\right)}}$

7. $\chi(Z)=c s t-Z \frac{F_{\bar{e}}(L)}{F_{\bar{e}}(H)} \frac{g\left(y_{H} \mid L, \bar{e}\right)-g\left(y_{H} \mid L, \underline{e}\right)}{g\left(y_{L} \mid L, \underline{e}\right)}$

8. $c s t=u\left[\frac{1}{F_{\bar{e}}(H)}-\frac{g\left(y_{L} \mid H, \bar{e}\right)}{g\left(y_{L} \mid H, \underline{e}\right)} \frac{F_{\bar{e}}(L) g\left(y_{L} \mid L, \bar{e}\right)}{F_{\bar{e}}(H) g\left(y_{L} \mid L, \underline{e}\right)}\right]+\frac{\Psi}{F_{\bar{e}}(H)}$

Hence, the principal will just maximize his objective function with respect to $Z$. Because the solution to Fudenberg and Tirole problem is valid only if $U(s, g, \bar{e}) \geq U(s, b, \bar{e})$, we are able to obtain - using the above equalities - a lower and an upper bound on $Z$. Let $U B$ and $L B$ be respectively the upper and lower bound on $Z$. They are defined by: $U\left(y_{H}, L, \bar{e}\right)(L B)=U\left(y_{L}, L, \bar{e}\right)(L B)$ $U\left(y_{H}, H, \bar{e}\right)(U B)=U\left(y_{L}, H, \bar{e}\right)(U B)$

We have the following properties:

$$
\begin{aligned}
& L B=\underset{L B \leq Z \leq U B}{\operatorname{Argmax}} U\left(y_{H}, H, \bar{e}\right)(Z)-U\left(y_{L}, H, \bar{e}\right)(Z)=\underset{L B \leq Z \leq U B}{\operatorname{Argmin}} U\left(y_{H}, L, \bar{e}\right)(Z)-U\left(y_{L}, L, \bar{e}\right)(Z) \\
& U B=\underset{L B \leq Z \leq U B}{\operatorname{Argmax}} U\left(y_{H}, L, \bar{e}\right)(Z)-U\left(y_{L}, L, \bar{e}\right)(Z)=\underset{L B \leq Z \leq U B}{\operatorname{Argmin}} U\left(y_{H}, H, \bar{e}\right)(Z)-U\left(y_{L}, H, \bar{e}\right)(Z)
\end{aligned}
$$

Furthermore, we have trivially that

$$
\begin{aligned}
& \frac{d\left(U\left(y_{H}, H, \bar{e}\right)(Z)-U\left(y_{L}, H, \bar{e}\right)(Z)\right)}{d Z}<0 \\
& \frac{d\left(U\left(y_{H}, L, \bar{e}\right)(Z)-U\left(y_{L}, L, \bar{e}\right)(Z)\right)}{d Z}>0
\end{aligned}
$$

Therefore, we have that $\mu(Z)<1$, for all $Z \in[L B, U B]$. Hence, if the principal decides to set the mixing probability strictly between $(0,1)$, the problem the principal 
faces is the following:

$$
\begin{aligned}
& \underset{L B \leq Z \leq U B}{\operatorname{Max}} \quad \mu(Z)\left\{g\left(y_{H} \mid \bar{e}\right) y_{H}+\left(1-g\left(y_{H} \mid \bar{e}\right)\right) y_{H} L\right. \\
& -F_{\bar{e}}(H)\left[g\left(y_{H} \mid H, \bar{e}\right) \Phi\left(U\left(y_{H}, H, \bar{e}\right)(Z)\right)+g\left(y_{L} \mid H, \bar{e}\right) \Phi\left(U\left(y_{L}, H, \bar{e}\right)(Z)\right)\right] \\
& \left.-F_{\bar{e}}(L)\left[g\left(y_{H} \mid L, \bar{e}\right) \Phi\left(U\left(y_{H}, L, \bar{e}\right)(Z)\right)+g\left(y_{L} \mid L, \bar{e}\right) \Phi\left(U\left(y_{L}, L, \bar{e}\right)(Z)\right)\right]\right\} \\
& \left.+(1-\mu(Z))\left\{g\left(y_{H} \mid \underline{e}\right) y_{H}+\left(1-g\left(y_{H} \mid \underline{e}\right)\right) y_{L}-\Phi(u)\right]\right\}
\end{aligned}
$$

subject to:

$$
\mu(Z)=\min \left\{\mu_{H}(Z), \mu_{L}(Z)\right\}
$$

There exists a solution to this problem as we maximize a continuous function over a compact set.

\subsection{Proofs}

\section{Proof of Lemma 3.2.}

We can rewrite $W_{1}$ as

$$
\begin{aligned}
W_{1}= & F_{\underline{e}}(H) \Phi(U(H))+\left(1-F_{\underline{e}}(H)\right) \Phi(U(L)) \\
& +\left[F_{\bar{e}}(H)-F_{\underline{e}}(H)\right][U(H)-U(L)] .
\end{aligned}
$$

We know that $\left[F_{\bar{e}}(H)-F_{\underline{e}}(H)\right][U(H)-U(L)]$ is non-negative and will show that $\Gamma \equiv$ $F_{\underline{e}}(H) \Phi(U(H))+\left(1-F_{\underline{e}}(H)\right) \Phi(U(L))$ goes to infinity as $F_{\bar{e}}(H) \longrightarrow F_{\underline{e}}(H)$. First note that $\Phi(U(H)) \longrightarrow \infty$ and $\Phi(U(L)) \longrightarrow-\infty$ as $F_{\bar{e}}(H) \rightarrow F_{\underline{e}}(H)$. Now,

$$
\begin{aligned}
\frac{\partial \Gamma}{\partial F_{\bar{e}}(H)}= & F_{\underline{e}}(H) \Phi^{\prime}(U(H))\left(-\Psi \frac{1-F_{\underline{e}}(H)}{\left[F_{\bar{e}}(H)-F_{\underline{e}}(H)\right]^{2}}\right) \\
& +\left(1-F_{\underline{e}}(H)\right) \Phi^{\prime}(U(L))\left(\Psi \frac{F_{\underline{e}}(H)}{\left[F_{\bar{e}}(H)-F_{\underline{e}}(H)\right]^{2}}\right) \\
= & \frac{\Psi}{\left[F_{\bar{e}}(H)-F_{\underline{e}}(H)\right]^{2}}\left[F_{\underline{e}}(H)\left(1-F_{\underline{e}}(H)\right)\right]\left[\Phi^{\prime}(U(L))-\Phi^{\prime}(U(H))\right]<0 .
\end{aligned}
$$

The last inequality holds by convexity of $\Phi$ and the fact that $U(H)>U(L)$. Also, by assumption, $F_{\bar{e}}(H)>F_{\underline{e}}(H)$ so as $F_{\bar{e}}(H)$ decreases, $\Gamma$ increases at an increasing rate and tends to infinity. 


\section{Proof of Lemma 3.4.}

From the expressions given above, we have that

$$
V_{\mu}-V_{0}=\mu\left[\underline{w}+\Delta\left(g\left(y_{H} \mid \bar{e}\right)-g\left(y_{H} \mid \underline{e}\right)\right)-W_{\mu}\right] .
$$

The proof follows since $W_{\mu} \geq 0$ and $\underline{w}+\Delta\left(g\left(y_{H} \mid \bar{e}\right)-g\left(y_{H} \mid \underline{e}\right)\right)$ is independent of $\mu$.

\section{Proof of Lemma 3.5.}

We can write

$$
V_{1}-V_{0}=\Delta\left[g\left(y_{H} \mid \bar{e}\right)-g\left(y_{H} \mid \underline{e}\right)\right]+\underline{w}-W_{1} .
$$

Notice that $W_{1}$ is independent of $\Delta$. The lemma holds as $g\left(y_{H} \mid \bar{e}\right)>g\left(y_{H} \mid \underline{e}\right)$.

\section{Proof of Theorem 4.1.}

(1) By lemma 3.2, $W_{1} \longrightarrow \infty$ as $F_{\bar{e}}(H) \longrightarrow F_{\underline{e}}(H)$. Our assumptions guarantee that $W_{\mu}$ is bounded so that for $F_{\bar{e}}(H)-F_{\underline{e}}(H)$ small enough, $V_{\mu}>V_{1}$.

(2) By lemma 3.5 we can choose $\Delta$ big enough so that $V_{1}^{\delta}\left(\mathcal{P}^{\prime}\right)>V_{0}$ holds. As $g\left(y_{H} \mid H, \bar{e}\right) \rightarrow$ $g\left(y_{H} \mid H, \underline{e}\right), U\left(y_{H}, H, \bar{e}\right) \rightarrow \infty$ and $U\left(y_{L}, H, \bar{e}\right) \rightarrow-\infty$. Therefore, given $\Phi^{\prime}>0$ and $\Phi^{\prime \prime}>0$, we have that $\Phi^{\prime}\left(U\left(y_{H}, H, \bar{e}\right)\right)-\Phi^{\prime}\left(U\left(y_{L}, H, \bar{e}\right)\right) \rightarrow \infty$. Hence, $\mu_{H}(Z) \rightarrow 0$, for all $Z$, and since $\mu=\min \left\{\mu_{H}(Z), \mu_{L}(Z)\right\}$, we have that $\mu(Z) \longrightarrow 0$ for all $Z$. Let $\varepsilon=\left(V_{1}(\delta)-V_{0}\right) / 2$. From lemma 3.4 we know that as $\mu \longrightarrow 0, V_{\mu}-V_{0}<\varepsilon$, so that $V_{1}>V_{\mu}$.

\subsection{Comparative statics with respect to $\Delta$ and $\Psi$}

In this appendix, we derive some of the intuitive results formally.

We have already proved that if $\Delta$ is big enough, $V_{1}>V_{0}$ and $V_{\mu}>V_{0}$. We also have:

Claim: There exists $\Delta>0$ small enough such that $V_{0}>V_{1}$ and $V_{0}>V_{\mu}$. 
Proof. Setting $\Delta=0$, we see that the claim holds. By continuity, and because $W_{1}$ and $W_{\mu}$ are independent of $\Delta$, the claim will hold for sufficiently small $\Delta>0$.

Claim: There exists $\Psi>0$ small enough such that $V_{1}>V_{0}$ and $V_{\mu}>V_{0}$.

Proof. As $\Psi \longrightarrow 0$, we have that $U(H)$ and $U(L)$ tend to $U$, such that $W_{1} \longrightarrow W_{0}$. Since $g\left(y_{H} \mid \bar{e}\right)>g\left(y_{H} \mid \underline{e}\right)$, there is a $\Psi$ low enough such that $V_{1}>V_{0}$. Similarly, for $V_{\mu}>V_{0}$.

Note that with $\Psi=0$, the principal will always choose $\mu=1$. But even for small $\Psi>0$, depending on the probabilities, it may be true that $V_{\mu}>V_{1}$.

Claim: There exists $\Psi>0$ large enough such that $V_{0}>V_{1}$ and, for any $\varepsilon>0$, $V_{\mu}-V_{0}<\varepsilon$.

Proof. We have $V_{1}-V_{0}=\Delta\left[g\left(y_{H} \mid \bar{e}\right)-g\left(y_{H} \mid \underline{e}\right)\right]+\Phi(u)-W_{1}$. The wage bill $W_{1}$ increases unboundedly with $\Psi$ and thus for $\Psi$ large enough, $V_{0}>V_{1}$.

Also, $V_{\mu}-V_{0}=\mu\left[\Phi(u)+\Delta\left(g\left(y_{H} \mid \bar{e}\right)-g\left(y_{H} \mid \underline{e}\right)\right)-W_{\mu}\right]$. As $\Psi$ increases, $W_{\mu}$ increases and $\mu$ decreases to zero.

\section{References}

[1] Azariadis, C., 1975. Implicit Contracts and Underemployment Equilibria. Journal of Political Economy 83, no. 6: 1183 - 1202.

[2] Azariadis, C., 1978. Escalator Clauses and the Allocation of Cyclical Risks. Journal of Economic Theory 18, no. 1: 119 - 155.

[3] Azariadis, C., and Cooper, R., 1985 a. Predetermined Prices and The Allocation of Social Risks. Quarterly Journal of Economics 100, no. : 495 - 518.

[4] Azariadis, C., and Cooper, R., 1985 b. Nominal Wage-Price Rigidity as a Rational Expectations Equilibrium. American Economic Review 75, no. 2: 31 - 35.

[5] Baily, M., 1974. Wages and Employment under Uncertain Demand. Review of Economic Studies 41, no. 125: 37 - 50. 
[6] Cooper, R., 1990. Predetermined Wages and Prices and the Impact of Expansionary Government Policy. Review of Economic Studies 57, no. 2: 205 - 214.

[7] Cukierman, A., 1984. Inflation, Stagflation, Relative Prices and Imperfect Information. Cambridge, Cambridge University Press.

[8] Fischer, S., 1977. Long-term Contracts, Rational Expectations and the Optimal Money Supply Rule. Journal of Political Economy 85, no. 1: 191 - 205.

[9] Freeman, S., and Tabellini, G., 1998. The optimality of nominal contracts. Economic Theory 11, no. 3: 545 - 562 .

[10] Fudenberg, D., and Tirole, J., 1990. Moral Hazard and Renegotiation in Agency Contracts. Econometrica 58, no. 6: 1279 - 1319.

[11] Gray, J., 1976. Wage indexation: A Macroeconomic Approach. Journal of Monetary Economics 2, no. 2: $221-235$.

[12] Jovanovic, B., and Ueda, M., 1997. Contracts and Money. Journal of Political Economy 105, no. 4: 700 - 708.

[13] Holland, S. A., 1995. Inflation and Wage indexation in the Postwar United States. Review of Economics and Statistics 77, no. 1: 172 - 176. 


\section{Figure 1}

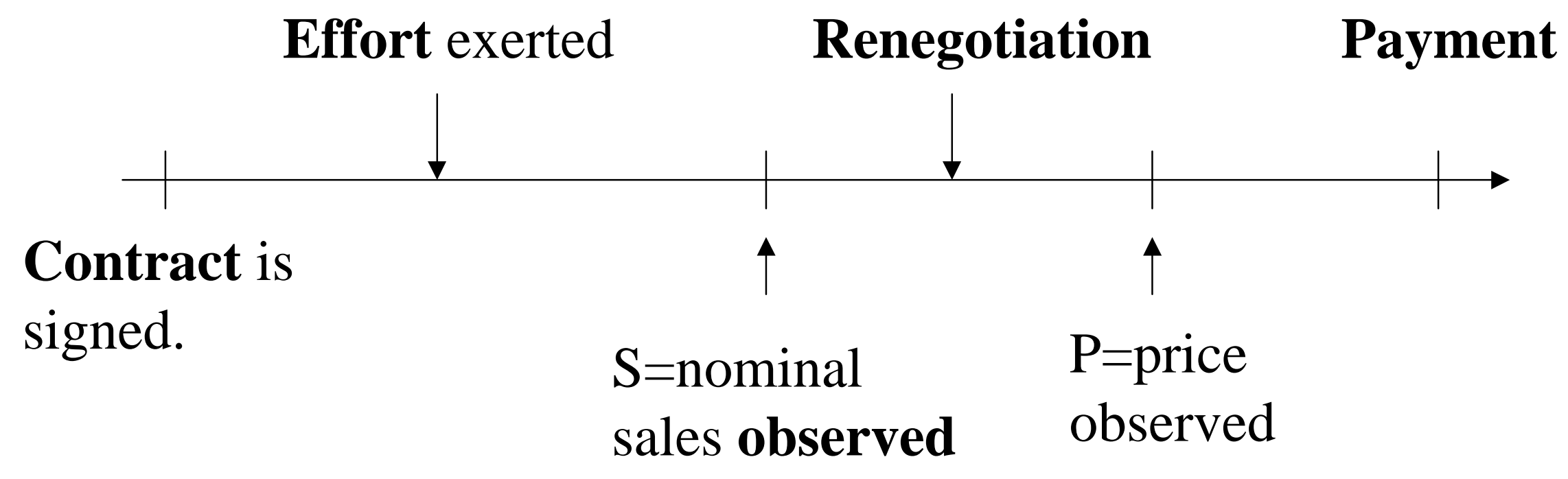

